\title{
A Novel Disease Caused by Increase of the Lifespan: Chronic Cutaneous Insufficiency Syndrome or Dermatoporosis
}

\author{
Badea MA ${ }^{1}$, Morariu $\mathrm{SH}^{2}$, Sin $\mathrm{Anca}^{3}$ \\ ${ }^{1}$ County Hospital of Tîrgu Mureș, Romania, Dermatology Clinic, Romania \\ 2 University of Medicine and Pharmacy TîrguMureș, Department of Dermatology, Romania \\ 3 University of Medicine and Pharmacy TîrguMureș, Romania, Department of Molecular and cell biology
}

Nowadays we are witnessing an increase in the medium lifespan caused by improved living conditions. The main factor in skin ageing is represented by the chronic exposure to ultraviolet radiation, however long term use of systemic or topical corticosteroids could produce similar effects on the skin. Chronic cutaneous insufficiency syndrome or dermatoporosis was described in 2007, being caused by a decreased activity of the hyaluronic acid, the main component of the extracellular matrix. This mechanism translates clinically through atrophy in the forearms or calves. Other injuries develop on this background including skin dissecting hematoma, which is a medical emergency. Therapeutic attitude consists of local application of preparations containing hyaluronic acid and retinaldehyde and discerning use of corticosteroid therapy. Photoprotection has a certain role in preventing the disease. While today dermatoporosis is a little known dermatosis even among dermatologists, in the future we will see a significant increase in the incidence of the disease and its gravity.

Keywords: dermatoporosis, elderly, sun exposure, corticotherapy

Received: 09 December 2014 / Accepted: 10 January 2015

\section{Introduction}

Medium lifespan doubled in most industrialized countries during the twentieth century. This has important repercussions in geriatric medicine by increased incidence of pathology and continuous emergence of new age-related diseases [1]. The main cutaneous ageing factor is the chronic exposure to ultraviolet radiation [2]. The term "chronic cutaneous insufficiency syndrome" was introduced in 2007 by two Swiss dermatologists, Kaya and Saurat. It is also commonly referred to as skin fragility syndrome or dermatoporosis. The key molecule involved in disease emergence is hyaluronic acid. [3]. The term was proposed due todermatoporosis generating mechanisms which are similar to osteoporosis.

\section{Clinical aspects}

Skin lesions occur in people aged 60 and is located in the forearms, more rarely in the calves. It is classified into four stages, from injuries that do not attract the attention of the patient and the clinician to the stage of skin dissecting hematoma (Table I). Initial lesions include atrophy, senile purpura and stellate pseudoscarrs. This dermatoporotic skin exposes the underlying epidermal structures. Subsequently, minimal trauma lead to skin ulceration or skin dissecting hematoma [3]. The latter are produced by the accumulation of blood between muscle fascia and subcutaneous fat. Skin lesions are similar to cellulite: painful er-

* Correspondence to: Silviu Horia Morariu

E-mail: silviu_morariu@yahoo.com ythemato-edematous plaques with increasedlocal temperature. They are seen in people over 80 years and are located in the calves. It is a life-threatening pathology, requiring emergency surgical drainage [4], [5].

Table I. Dermatoporosis stages

\begin{tabular}{lccc}
\hline \multicolumn{2}{c}{ Dermatoporosis } \\
\hline Stage I & $\begin{array}{c}\text { Atrophy } \\
\text { Stellate } \\
\text { pseudoscars } \\
\text { Senile Purpura }\end{array}$ & \\
Stage II & & Skin lacerations & \\
Stage III & & Skin lacerations + & \\
Stage IV & & Skin lacerations ++ & Deep Dissecting \\
& & & Hematomas \\
\hline
\end{tabular}

\section{Molecular mechanisms}

The main component of the extracellular matrix between the keratinocytes is the hyaluronic acid (HA). It is the main molecule that is affected in case of skin aggression, whether physical, chemical or mechanical. This glycosaminoglycan binds to the surface of the keratinocytes through the CD 44 receptor. This coupling initiates a series of molecular signals which have a role in proliferation, differentiation and cell migration [6]. Expression of the HA and of the $\mathrm{CD} 44$ receptor is diminished by ultraviolet radiation type $\mathrm{A}$ and $\mathrm{B}[7]$.

Molecular mechanisms of chronic cutaneous insufficiency are based on a reduction in hyaluronic acid activity due to diminished expression of CD44 receptors on the surface of keratinocytes [8]. A decreased activity of the 


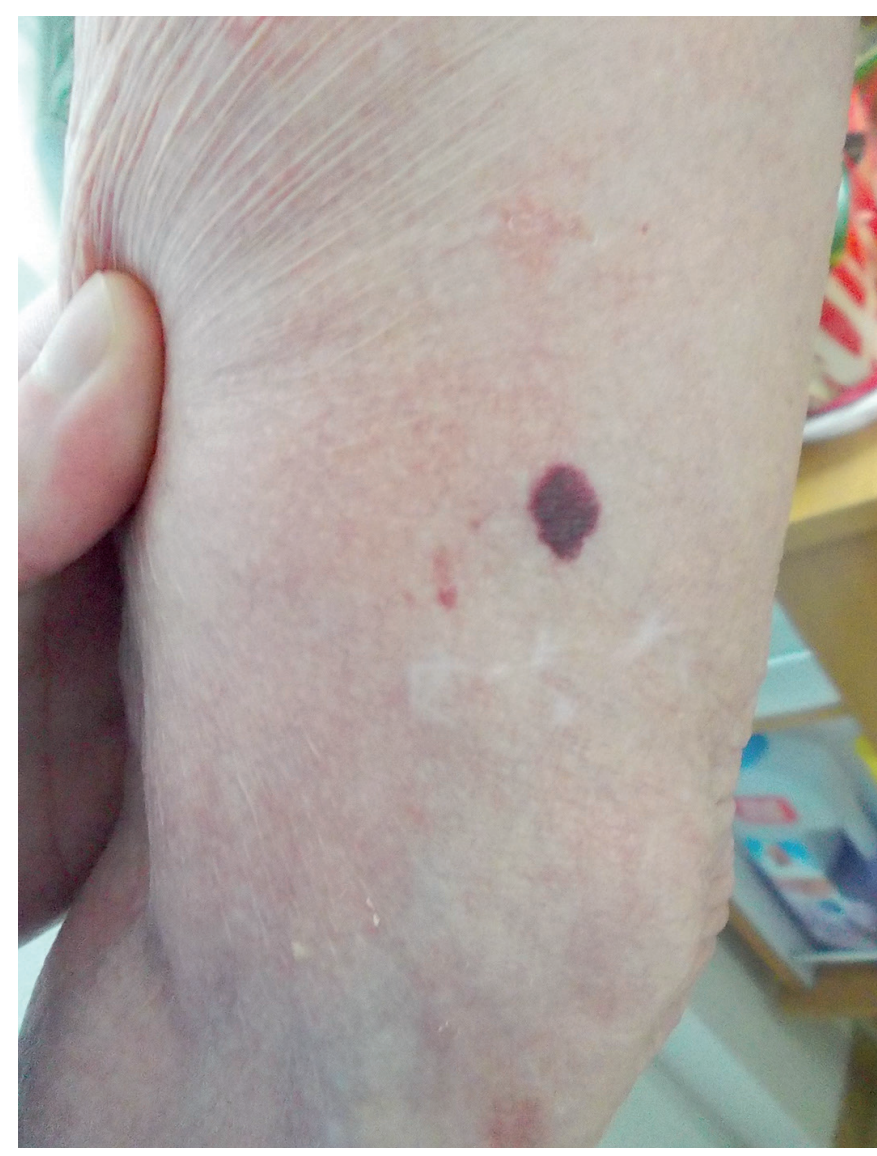

Fig. 1. Dermatoporosis on forearms in a female patient with psoriasis who was treated with topical clobetasol propionate for 8 years

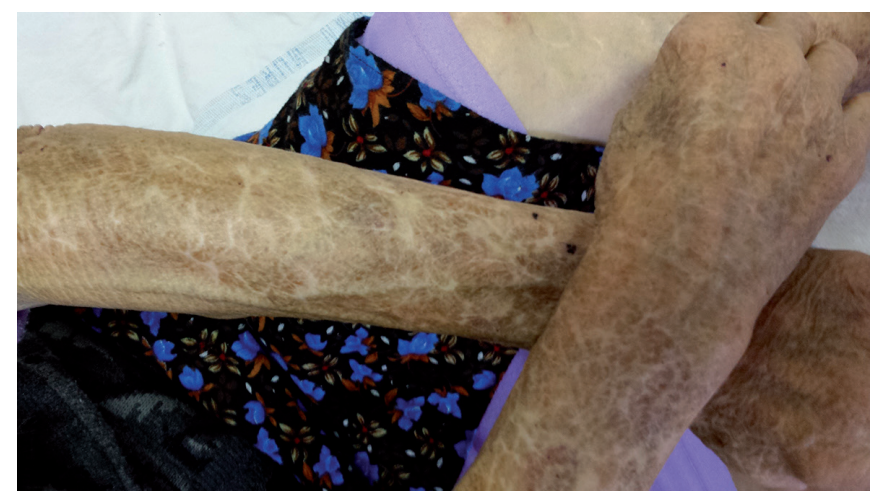

Fig. 3. Dermatoporosis on forearms in a elderly female (92 years old) with chronic sun exposure.

hyaluronic acid synthase (HAS) and a decreased expression of the epidermal growth factor receptor (EGFR) are also present. A possible role of the activation of metalloproteinase-7 with a possible role in extracellular matrix degradation is discussed [3], [9], [10].

\section{Therapeutic attitude}

Based on molecular aspects, the treatment of choice is the topical application of preparations containing hyaluronic acid. Recently a therapeutic superiority was demonstrated by adding retinaldehyde. This causes increased expression of CD 44 receptor [11]. These events should be known by all clinicians in order to correct therapeutic attitude.

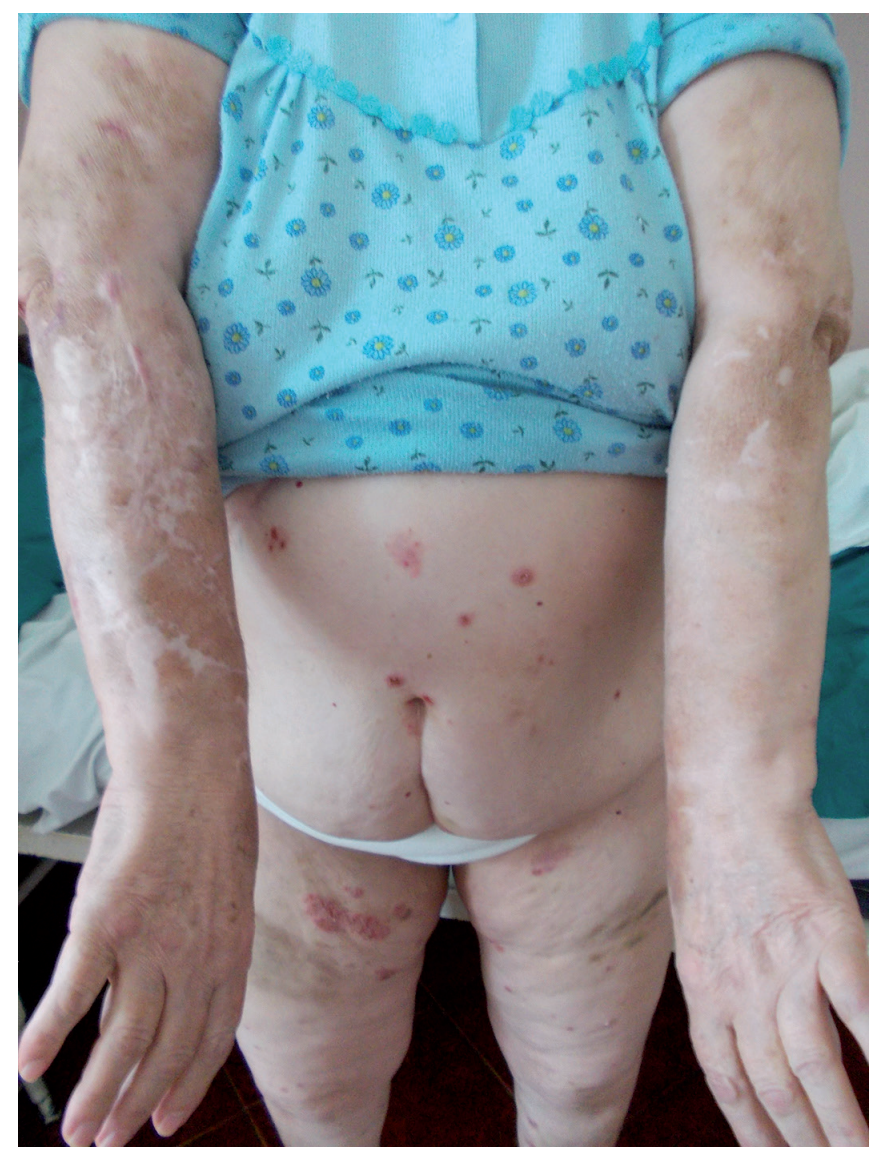

Fig. 2. Dermatoporosis on forearms in a female patient who received long term corticotherapy for Bullous pemphigoid

This consists of avoiding local corticosteroid and discerning use of the systemic, application of creams containing hyaluronic acid and retinaldehyde, using the methods of photoprotection. Indeep dissecting hematoma (DDH) the therapeutic attitude consists in their emergency drainage.

\section{Discussions}

Although the areas most exposed to solar radiation are the face, back of hands and nuchal region, dermatoporosis lesions occur almost exclusively on the forearms and less often on the calves. It seems that this is due to the skin structure at this level, being thinner than in other areas. This causes atrophy development, on which other injuries overlap.

Dermatoporotic skin appearance was described 40 years ago and was framed with other dermatoses characterized by atrophy under the generic term of atrophoderma [12].

Kurashige et al describe the association of primary dermatoporosis with sacral pressure ulcer in a series of three patients aged over 70 years. These cases may demonstrate the general fragility of the cutaneous structure, the clinical expression being triggered by a mechanical factor. We cannot exclude the association of these two specific dermatoses, without a direct causal relationship between them [13].

Yoneda et al. found a direct association between the degree of atrophy of the skin on the forearms and the degree 
Table II. Therapeutic attitude in dermatoporosis

\begin{tabular}{l} 
Therapeutic measurements in dermatoporosis \\
\hline Topical hyaluronic acid and retinaldehid \\
Avoid systemic or topic corticotherapy \\
Use photoprotection methods \\
Protect the area from mechanical injuries
\end{tabular}

Table III. Therapeutic attitude in deep dissecting hematoma

\begin{tabular}{l} 
Deep Dissecting Hematomas \\
\hline Dermatoporosis signs on shins and/or forearms \\
Edematous patch/plaque or erythema on the shins with local increase in \\
temperature and local pain present \\
Absence of fever \\
MRI- bright band between subcutaneous fat and adjacent muscle \\
Surgical drainage followed by primary suture/skin graft/per secundam \\
healing
\end{tabular}

of osteoporosis in women during menopause. The degree of osteoporosis was quantified by bone densitometry. Cutaneous atrophy was measured with skinfold calipers, which may lead to measurement errors, being a clinical, investigator- dependent method, which provides equivocal data. The quality of skin atrophy measurement would significantly increase by using a skin ultrasound.

The use of molecular parameters for quantifying the degree of degradation of the skin forearms such as determining the metaloproteinase- 7 by immunohistochemistry or the epidermal atrophy by histopathology would have been more more specific. Such studies are needed to demonstrate the parallel evolution of osteoporosis and dermatoporosis, assuming that both have the mechanism of a decrease in the amount and activity of hyaluronic acid [14]. Induction of skin atrophy after applying topical corticosteroid was demonstrated by Dykes et al since 1976. They describe a decrease in epidermal thickness on the forearm following the application of Fluocinoloneacetonide in human subjects. Atrophy was quantified by measuring with Skinfold Caliper [15].

Local application of potent topical corticosteroids has the same clinical, histopathological and molecular effects as chronic exposure to ultraviolet radiations. Burnes et al. demonstrated in a study on mice a disruption in hyaluronic acid function and of the epidermal growth factor (EGF) after applying clobetasol propionate. These changes translate into a decreased number of CD 44 receptors, a decreased activity of hyaluronic acid synthase (HAS) and of the epidermal growth factor receptor(EGFR) [16].

Deep Dissecting hematoma are specific for final stage dermatoporosis. Kaya et al found that women were more affected (W/ $/ M=5: 1$ ) with a mean age of 81.7 years. In their study, all 34 patients presented these hematomas in the anterior calves. They were developed amid dermatoporosis advanced lesions. They showed signs of erysipelas or cellulitis but without fever [4].In the presence of these clinical signs and knowing the signs of dermatoporosis, es- pecially in the case of elderly patients a diagnosis of DDH should be suspected. This is also confirmed by MRI which shows a hyperechoic band between subcutaneous fat and muscle adjacent structures. Therapeutic attitude consists of hematoma drainage followed by primary suture, skin graft or healing per secundam [5].

\section{Conclusions}

Dermatoporosis occurs in the elderly and is located on the forearms or calves. The treatment of choice is the topical application of preparations containing hyaluronic acid and retinaldehyde.

Deep Dissecting Hematomas represent the final stage of dermatoporosis and occur only in the calves. Diagnosis is by MRI and require emergency surgical drainage.

\section{References}

1. Sander M, Oxlund B, Jespersen A et al. The challenges of human population ageing. Age Ageing.2014 Dec 1.pii: afu189. [Epub ahead of print]

2. Helfrich YR, Sachs DL, Voorhees JJ: Overview of Skin Aging and Photoaging. Dermatology Nursing. 2008;20(3):177-183.

3. Kaya G, Saurat JH.Dermatoporosis: a chronic cutaneous insufficiency/ fragility syndrome. Clinicopathological features, mechanisms, prevention and potential treatments. Dermatology. 2007;215(4):284-294.

4. Kaya G, Jacobs F, Prins C, etal.Deep dissecting hematoma: an emerging severe complication of dermatoporosis. Arch Dermatol. 2008;144(10):1303-1308.

5. Gamo R, Vicente J, Calzado L, et al.: Deep Dissecting Hematoma or Stage IV Dermatoporosis. ActasDermosifiliogr. 2010;101(1):89-106.

6. Maytin EV, Chung $\mathrm{HH}$, Seetharaman VM et al. Hyaluronan participates in the epidermal response to disruption of the permeability barrier in vivo. Am J Pathol. 2004;165(4):1331-1341.

7. Calikoglu E, Sorg O, Tran C, et al. UVA and UVB decrease the expression of CD44 and hyaluronate in mouse epidermis, which is counteracted by topical retinoids. PhotochemPhotobiol. 2006;82(5):1342-1347.

8. Kaya G, Saurat JH. Dermatoporosis: A new concept in skin aging. European Geriatric Medicine.2010;1(4):216-219.

9. Saurat JH: Editorial.Quand la peaudevientinsuffisante.Med Hyg.2004; 2472-2476.

10. Saurat JH: Editorial: Dermatoporosis: The functional side of skin aging. Dermatology 2007; 215: 271-272.

11. Nikolic DS, Ziori C, Kostaki $M$ et al. Hyalurosome gene regulation and dose-dependent restoration of skin atrophy by retinaldehyde and defined-size hyaluronate fragments in dermatoporosis. Dermatology. 2014;229(2):110-115.

12. Piérard-Franchimont C, Hermanns JF, Hermanns-Lê $T$ et al. Dermatoporosis, a vintage for atrophoderma and transparent skin. Rev Med Liege. 2014;69(4):210-213.

13. Kurashige Y, Minemuta T, Nagatani T.Three Cases of Sacral Pressure Ulcers Presenting Primary Dermatoporosis on the Forearms. Case Rep Dermatol, 2013;5(1):73-78.

14. Yoneda PP, Biancolin SE, Gomes MSM et al. Association between skin thickness and bone density in adult women. An Bras Dermatol.2011;86(5):878-884.

15. Dykes PJ, A Francis AJ, Marks R. Measurement of dermal thickness with the harpenden skinfold caliper. Archives of Dermatological Research. 1976;256(3): 261-263.

16. Barnes L, Ino F, Jaunin F et al. Inhibition of Putative Hyalurosome Platform in Keratinocytes as a Mechanism for Corticosteroid-Induced Epidermal Atrophy. Journal of Investigative Dermatology. 2013;133:1017-1026. 